دراسة تأثير انحراف الفرن الدوار عن محور العجلات الحاملة في معامل الأسمنت على الألى الإجهادات في المحامل مئل

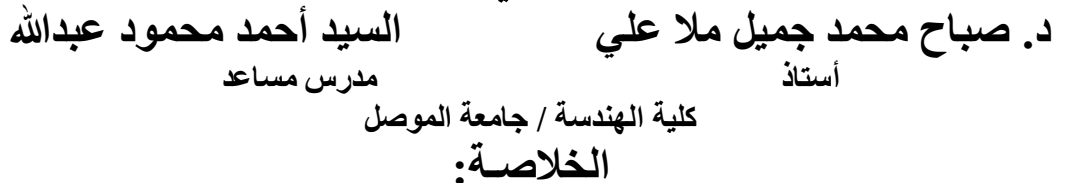

تعد مثكلة عدم المحاذاة في الأفران الدوارة (Rotary Kilns) من أهم المثاكل التشاصة التشغيلية الثائعة والمتكررة

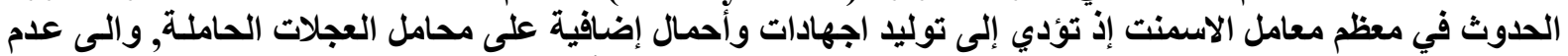

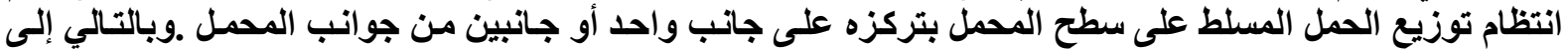

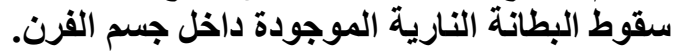

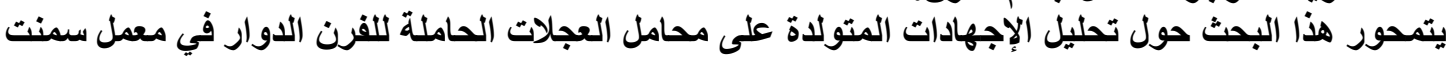

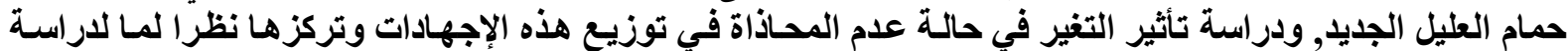

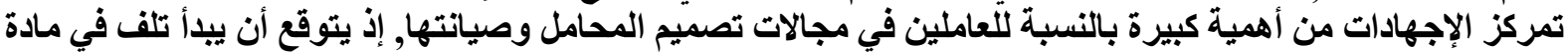
المحامل عند مناطق تركز الإجهادات.

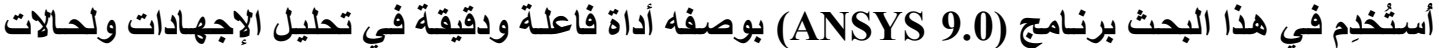

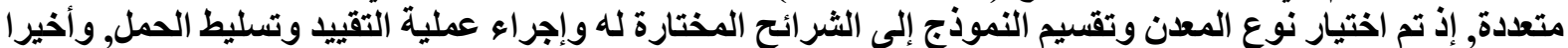

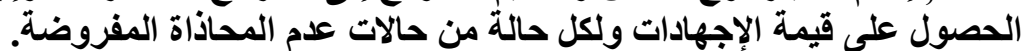

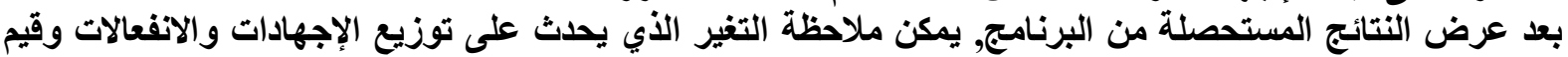

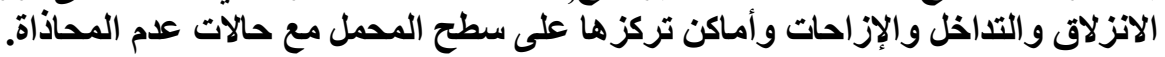

\title{
Study of the Misalignment of the Rotary Kiln Relative to Carrying Roller Center in Cement Factories on the Stress in Bearings
}

\author{
Dr. Sabah. m. j. Ali \\ Professor \\ Mr. Ahmed M. Abdullah \\ Assistant Lecturer \\ College of Engineering / University of Mosul
}

ABSTRACT:

Misalignment problem is considered as one of the most important and common repeated problems in rotary kiln in meanly all of cement factories. This will lead to generation of vibration and extra loads on bearing of the carrying rollers. Also a nonuniformity can occur in distribution of applied load on all the surface of the bearing which being concentrated on one or two sides of the bearing surface, and finally leads to falling the fire lining.

The present research work is concentrated on the analysis of stresses generated on bearing of the carrying rollers of the rotary kiln in new Hammam Al-Alil factory. In addition the effect of misalignment is shown on stresses distribution and it's concentration. This is important for the bearing design and those who work in bearing maintenance in this factory.

The "(ANSYS 9.0)", which uses a finite element technique, had been utilized in this program. This is a very efficient and accurate tool in stress analysis for many cases. The stresses had been calculated for the assumed cases.

The results showed clearly that the stress distribution and concentration on the bearing is altered with misalignment for each case.

Keywords: Misalignment, Rotary kiln, Techniques, ANSYS

$$
\text { قبل: } 2010 \text { - } 90
$$$$
\text { أستلم: } 2010 \text { - } 2 \text { - } 26
$$ 
تعريف الرموز والمصطلحات:

\begin{tabular}{|c|c|c|c|c|c|}
\hline الوحدة & تعريفه & الرمز & الوحدة & تعريفه & 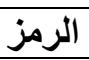 \\
\hline (Degree) & زاوية ميلان المستوي & $\phi$ & $\mathrm{kN} / \mathrm{m}^{2}$ & معامل يونج للمرونة. & $E$ \\
\hline $\mathrm{kN} / \mathrm{m}^{2}$ & الإجهاد العمودي & $\sigma$ & - & مسند الفرن الأول & I \\
\hline $\mathrm{kN} / \mathrm{m}^{2}$ & الإجهاد باتجاه المحور (z). & $\sigma_{z}$ & - & مسند الفرن الثاني & II \\
\hline$k N / m^{2}$ & الإجهاد الأساسي الأعظم & $\sigma_{1}$ & - & مسند الفرن الثالث & III \\
\hline $\mathrm{kN} / \mathrm{m}^{2}$ & الإجهاد الأساسي الأدنى & $\sigma_{2}$ & - & مسند الفرن الر ابع & IV \\
\hline$k N / m^{2}$ & الإجهاد باتجاه المحور (y) & $\sigma_{y}$ & - & مسند الفرن الخامس & $\mathrm{V}$ \\
\hline$k N / m^{2}$ & إجهاد القص & $\tau$ & $\mathrm{kN}$ & القوى باتجاه المحور (x) & $\mathrm{Fx}$ \\
\hline$(\mathrm{kN})$ & رد الفعل للجهة اليسرى & $\mathrm{R} 2$ & $\mathrm{kN}$ & القوى باتجاه المحور (y) & Fy \\
\hline & & & $(\mathrm{kN})$ & رد الفعل للجهة اليمنى & $\mathrm{R} 1$ \\
\hline
\end{tabular}

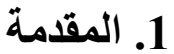

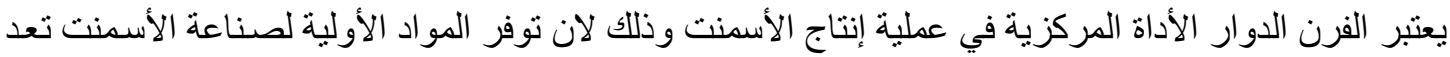

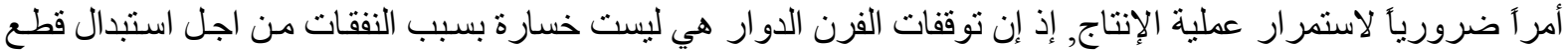

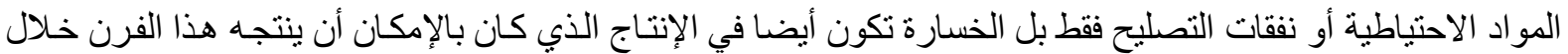

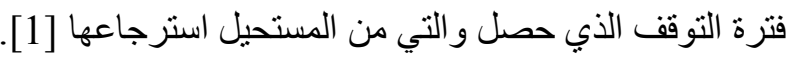
أجريت عدد من البحوث على الأفران الدوارة, حيث قام الباحثان Maged S.A , Sultan J.N) ) في مكتب التب

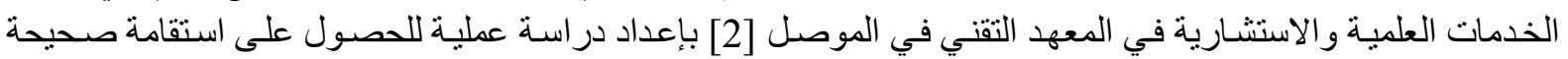

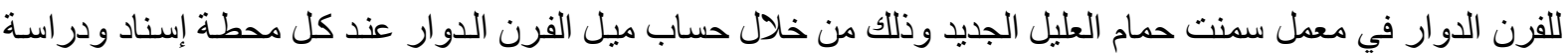

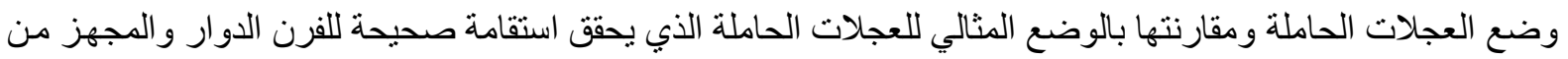

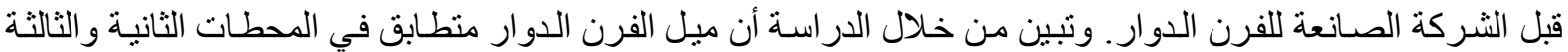

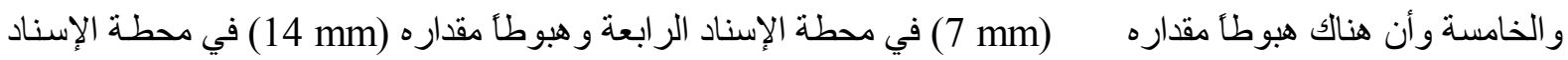

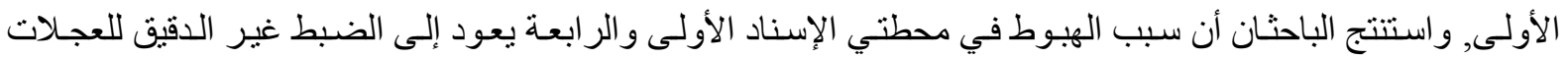

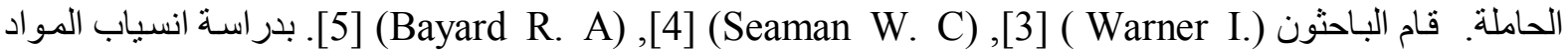

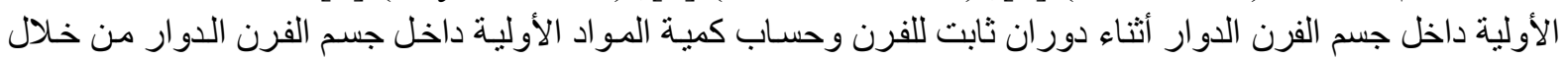

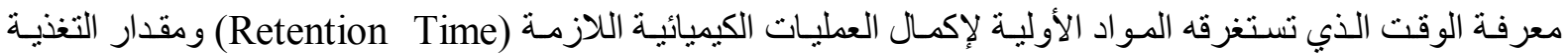

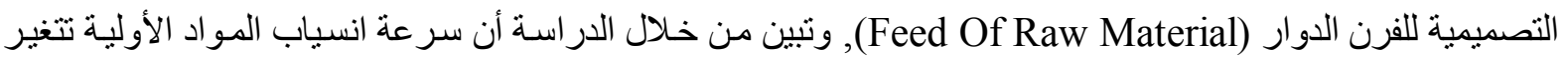

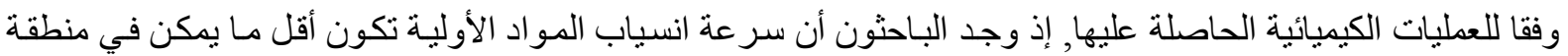

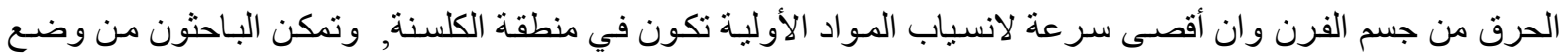

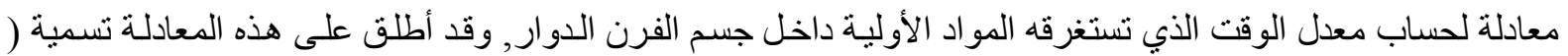

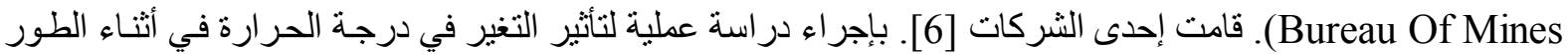

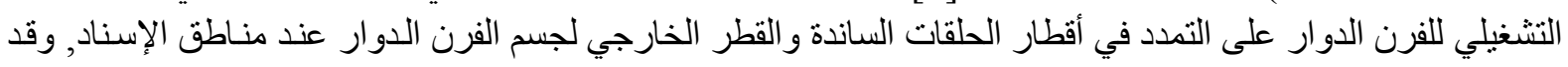

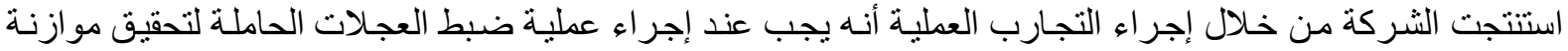

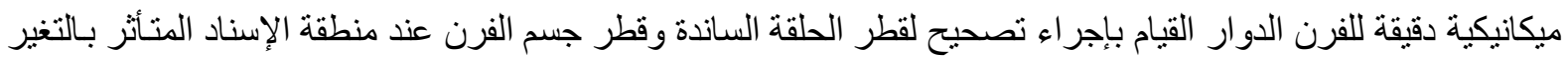

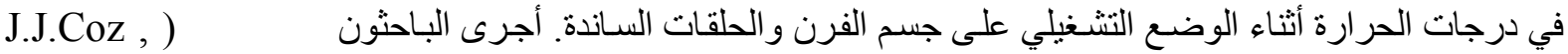
(F.Rodriguez and P.J Garcia في الجزائر, وذللك باستخدام تقنية العنصر المحدد (Rais Hamidou) اللاخطي للاجهادات المتولدة على جسم الفرن الدوار و الناتج عن التلامس بين جسم الفرن والحلقات الساندة, إذ تم بنـاء

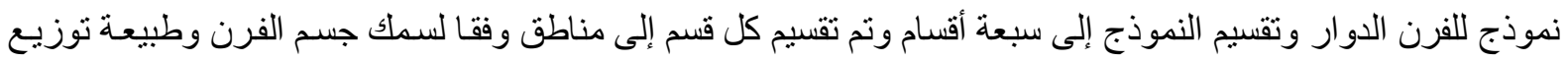




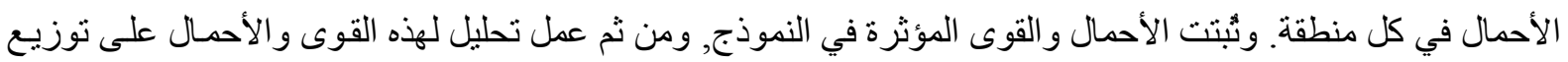

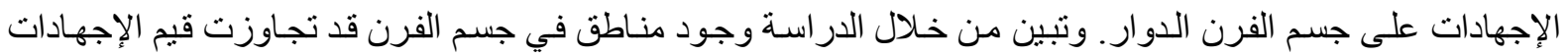

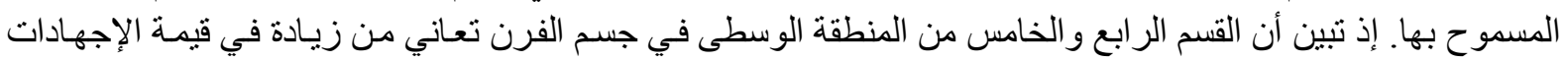

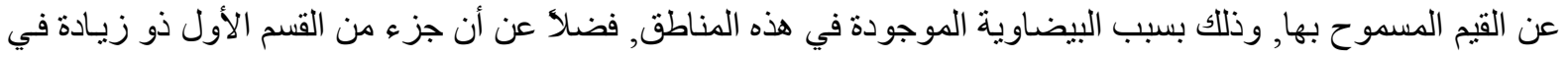

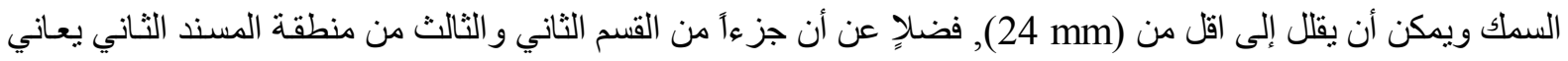

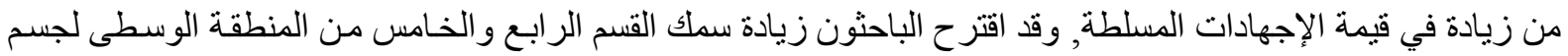

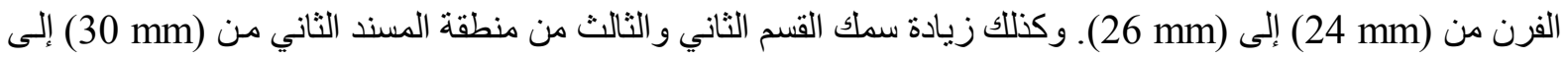
(40mm)

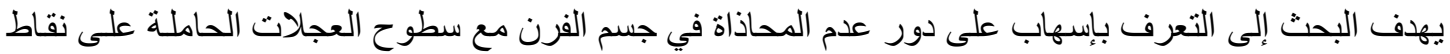

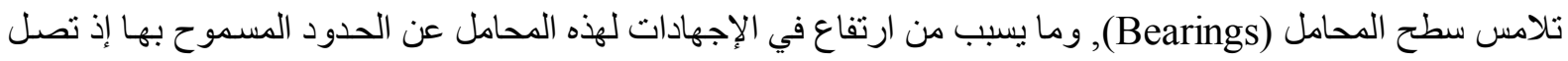

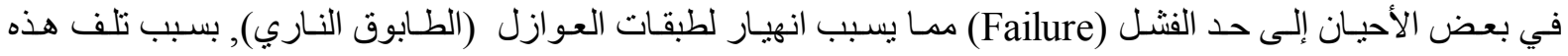

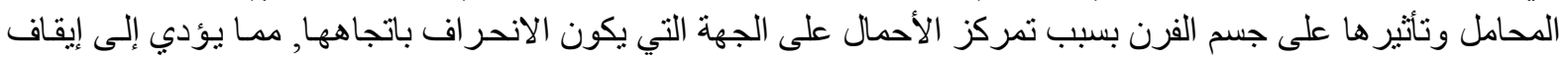

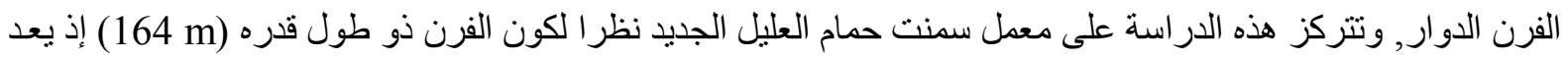

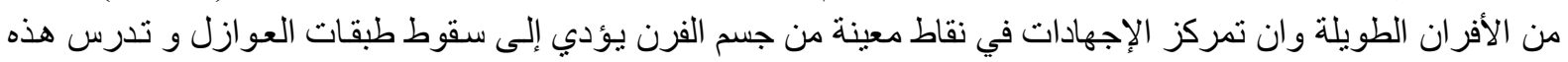

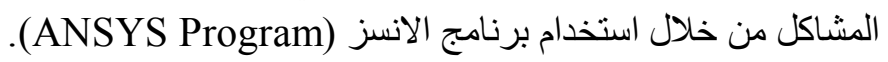

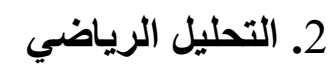

تم حساب الأحمال المؤثرة على جسم الفرن الدوار في المعمل وذللك من خلال معرفة طبيعة تلك الأحمال و أسلوب توزيعها

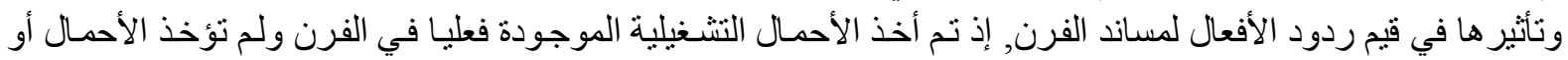

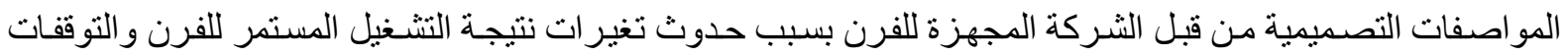

المفاجئة له.

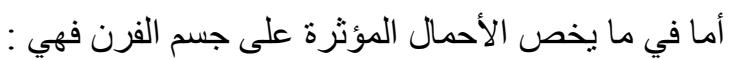

1 - وزن جسم الفرن الدوار (Weight of Kiln Shell).

2- وزن الحلقات الساندة (Weight of Live Rings).

3- وزن البطانة النارية (Weight of Refractory)

4- وز وزن النرس الكبير (Weight of Girth Gear).

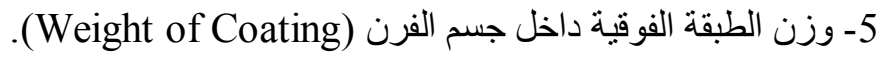

6- وز وزن المو اد الأولية داخل الفرن دان (Weight of Raw Materials)

7- وزن بلاط المخرج (Weight of Kiln Nose Ring).

8- وزن سلاسل التبادل الحر اري داخل جسم الفرن (Weight of Chains).

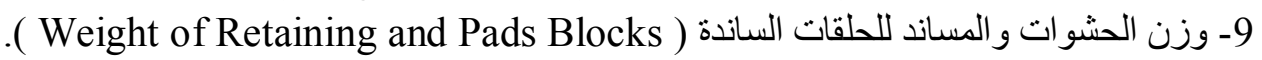

10- وزن جيوب التبادل الحراري داخل جسم الفرن (Weight of Buckets).

وفيما ينعلق بقيم الحمل الكلي وحسب موقعه فهذا مبين في الجدول (1) التالي

الجدول (1): حساب الحمل الكلي لكل منطقة من مناطق الفرن.

\begin{tabular}{|c|c|c|c|c|c|c|}
\hline V - Inlet & IV - V & III - IV & II - III & I - II & Outlet - I & الموقع الكمل \\
\hline $22899 \mathrm{~kg}$ & $423019 \mathrm{~kg}$ & $570571 \mathrm{~kg}$ & $560695 \mathrm{~kg}$ & $594419 \mathrm{~kg}$ & $86513 \mathrm{~kg}$ & \\
\hline
\end{tabular}




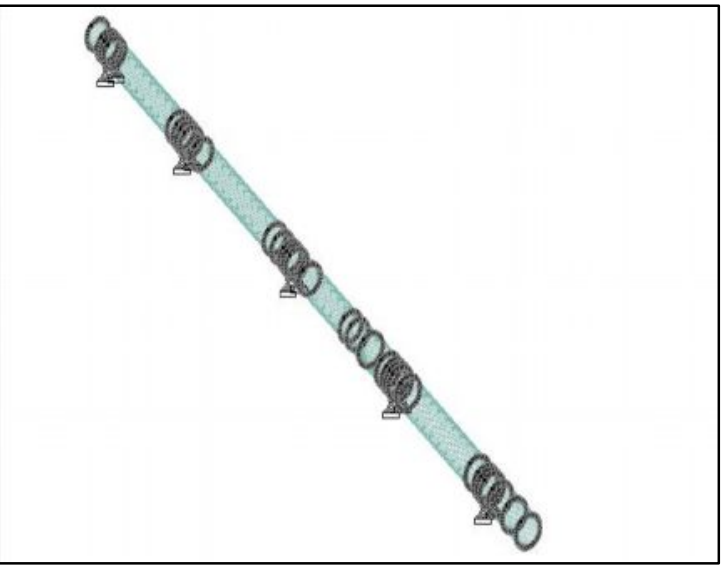

الثكل (1): نموذج الفرن الدوار باستخدام برنامج

.Staad Pro 2004

3. الأسلوب المتبع لإيجاد قيم ردود الأفعال

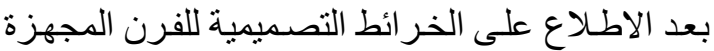

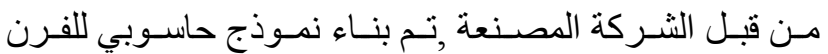
باستخدام برنامج (Staad Pro 2004), إذ تم بناء النموذج و

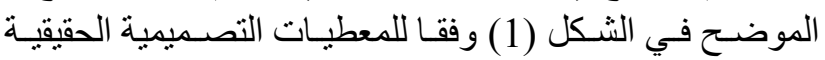
للفرن من حيث الثكل والأبعاد.

وبعد الانتهاء من بناء النموذج للفرن جرت عملية

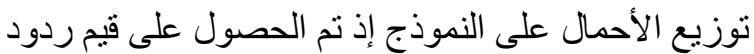

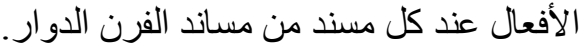

أنُبِعَت الفرضبات في عملية بناء النموذج و إيجاد قيم

ردود الأفعال على مساند الفرن وان هذه الفرضيات هي [8,9].

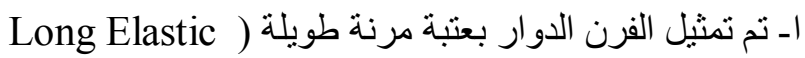
Beam

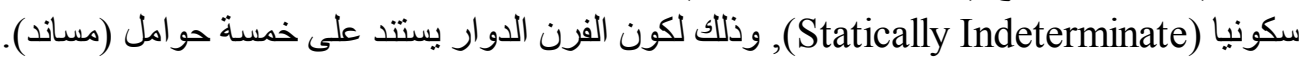

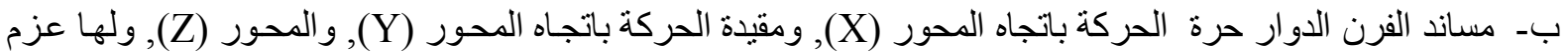

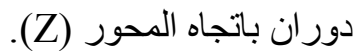
جـ- إهمال تأثير دوران الفرن أي اعتبار الفرن في طور التوقف إذ إن السرعة الدورانية للفرن قليلة, حيث انه لا تتجاوز ألـ (1.p.m)

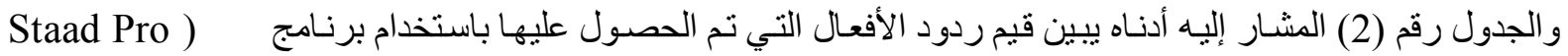
الجدول (2): يوضح قيم ردود الأفعال على مساند الفرن.

\begin{tabular}{|c|c|c|}
\hline \hline Kiln Supporting Number & Fx $(\mathrm{kN})$ & Fy $(\mathrm{kN})$ \\
\hline I & 60.42 & 3081.2 \\
\hline II & -14.1 & 6006.7 \\
\hline III & -6.1 & 6219.8 \\
\hline IV & 3.1 & 5287.8 \\
\hline V & 0.7 & 4237.8 \\
\hline
\end{tabular}

وباستخدام متلث القوى الشكل (2), تم إيجاد قيم ردود الأفعال عند كل عجلة حاملة من عجلات الفرن الدوار

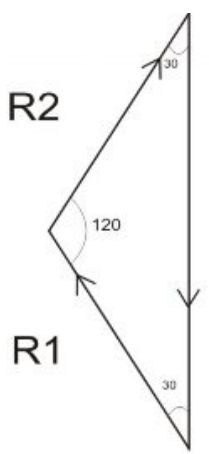

Fy

الثكل (2): منلث القوى الذي يوضح طريقة حساب ردود الأفعال عند كل عجلة حاملة من عجلات الفر ن الدو ار

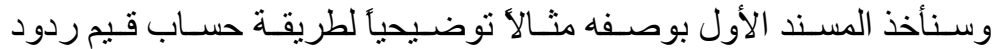
الأفعال.

حيث أن: = Fy

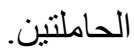
= $R_{1}$

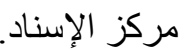
= R2

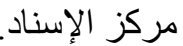
أما بقية العجلات الحاملة فقد نم احتساب قيم ردود الأفعال بالطريقة نفسهاو وضعها في الجدول رقم (3). 
الجدول (3): يوضح قيم ردود الأفعال على العجلات الحاملة للفرن.

\begin{tabular}{|c|c|c|}
\hline \hline Kiln Supporting Number & R1 ( kN ) & R2 ( kN ) \\
\hline I & 1779 & 1779 \\
\hline II & 3468 & 3468 \\
\hline III & 3591 & 3591 \\
\hline IV & 3053 & 3053 \\
\hline V & 2447 & 2447 \\
\hline
\end{tabular}

4. الإجهادات المركزية في حالات التلامس فلاكس

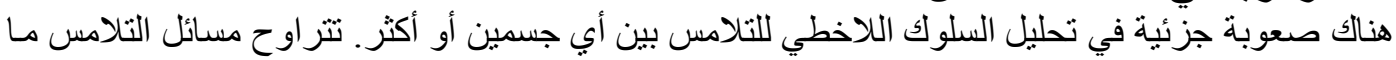

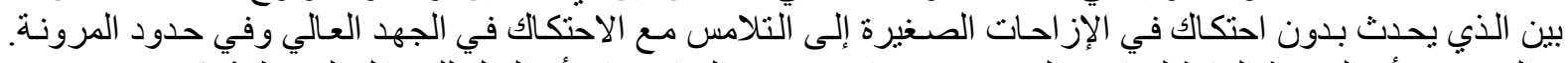

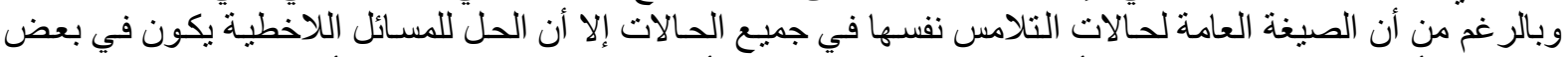
التحليلات أكثر صعوبة من حالات أخرى, ومن الجدير بالذكر أن اللاخطية في تحليل الدسألة هي التي تحدد حسالات

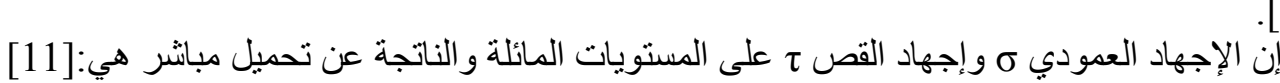

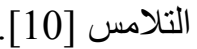
$\tau=-\frac{1}{2} \sigma_{y} \sin 2 \phi$

$\sigma=\sigma_{y} \sin ^{2} \phi$

و ان الإجهادات المسلطة على المستويات المائلة والناتجة من نظم إجهادات معقدة هي :

Normal stress $=\frac{1}{2}\left(\sigma_{x}+\sigma_{y}\right)+\frac{1}{2}\left(\sigma_{x}-\sigma_{y}\right) \cos 2 \phi+\tau_{x y} \sin 2 \phi$

Shear stress $=\frac{1}{2}\left(\sigma_{x}-\sigma_{y}\right) \sin 2 \phi-\tau_{x y} \cos 2 \phi$

Normal stress وان الاجهادات Shear stress و إن الإجهادات الرئيسة (أي الإجهادات العظمى المباشرة و الإجهادات الدُنيا المباشرة) و الني تقع في مستوي تكون فيه $\sigma_{1}=\frac{1}{2}\left(\sigma_{x}+\sigma_{y}\right)+\frac{1}{2} \sqrt{\left[\left(\sigma_{x}-\sigma_{y}\right)^{2}+4 \tau_{x y}^{2}\right]}$

$\sigma_{2}=\frac{1}{2}\left(\sigma_{x}+\sigma_{y}\right)-\frac{1}{2} \sqrt{\left[\left(\sigma_{x}-\sigma_{y}\right)^{2}+4 \tau_{x y}^{2}\right]}$

وإن هذه الإجهادات تحدث فوق مستويات وبزاوية $\phi$ نسبة إلى المستوي الذي يؤثر $\sigma_{x}$ فيها, إذ إن: $\tan 2 \phi=\frac{2 \tau_{x y}}{\left(\sigma_{x}-\sigma_{y}\right)}$

$\tan \phi=\frac{\sigma_{p}-\sigma_{x}}{\tau_{x y}}$

إذ إن 
في الغالب المستويات الرئيسة تميل بزاوية $90^{\circ}$ على كل منهما, بينما تميل مستويات القص بزاوية $45^{\circ}$

$$
\tau_{\text {max }}=\frac{1}{2} \sqrt{\left[\left(\sigma_{x}-\sigma_{y}\right)^{2}+4 \tau_{x y}^{2}\right]}=\frac{1}{2}\left(\sigma_{1}-\sigma_{2}\right)
$$

و في المسائل التي يكون فيها الإجهاد الرئيس في محور ثالث فإن م3 تكون دائما ذات قيمة معلومـة أو تفرض مساوية للصفر , وإن إجهاد القص الأعظم الحقيقي يساوي: إجهاد القص الأعظم الحقيقي= $\frac{1}{2}$ (الإجهاد الرئيس الأعظم ـ الإجهاد الرئيس الأصغر ) و إن الإجهاد العمودي على المستويات ذات القص الأعظم = و إن إجهاد القص على المستوي ذات الإجهاد الأعظم المباشر [11, 12] (المسنوي الرئيس) = 0.

1- Rigid - to - Flexible.

و فيما يخص التصنيف العام للنتامس فهي تقع ضمن التصنيفين العامين التالين [10].:

2- Flexible - to - Flexible.

$$
\text { جاسيء - إلى - مرن - مرن }
$$

في حالة الجاسيء (Rigid) هو الذي يكون أكثر صلادة بالنسبة للجسم القابل للتشويه (سطح الجسم الثناني و الملامس لسطح الجسم الأول).

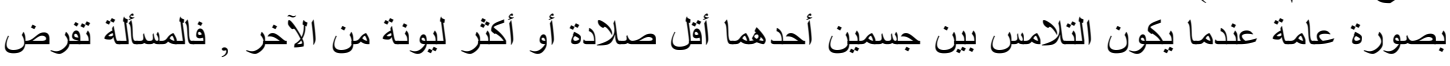

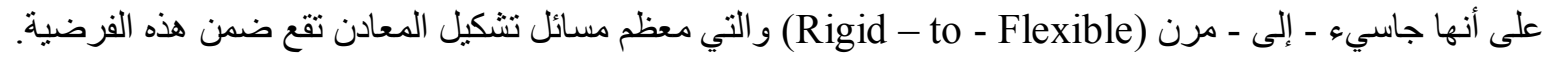

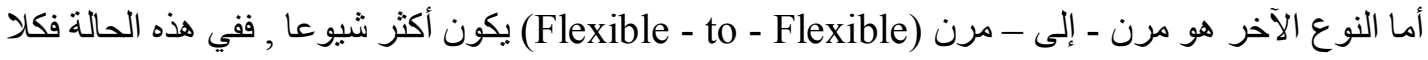

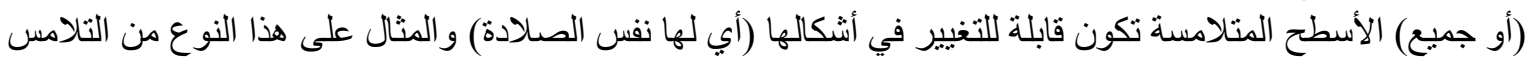

هو الثفة الملولبة (Bolted Flange).

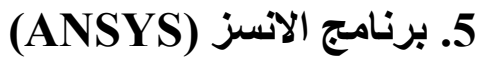

\subsection{SOLID95 - 3-D 20-Node Structural Solid}

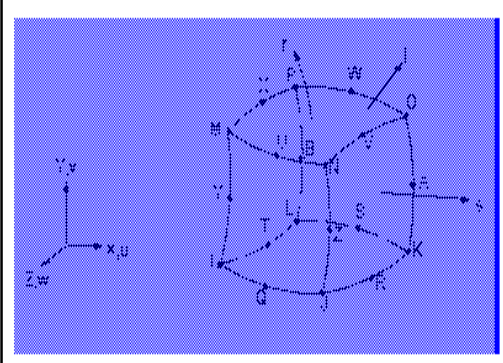

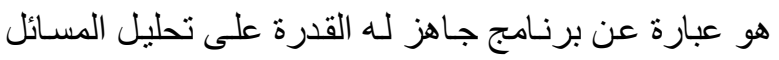

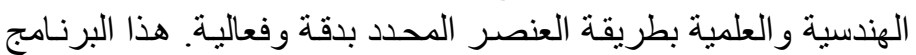

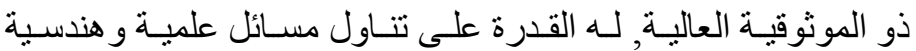

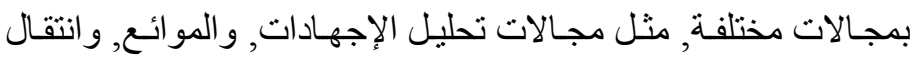

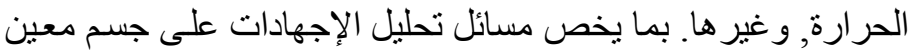

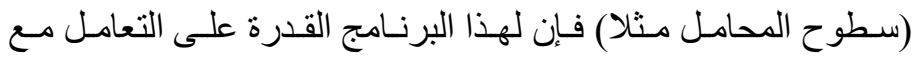

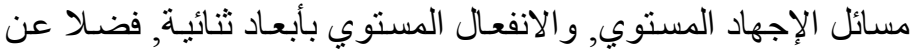

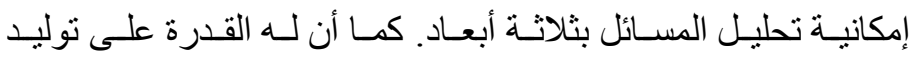

الثكل (3): عنصــر ثلاثــي الأبعــاد سداسي الأوجه ذو عشرين عقدة عند
التقسيمات ذاتيا بعد تحديد نوع العنصر ودرجة التقسيم.

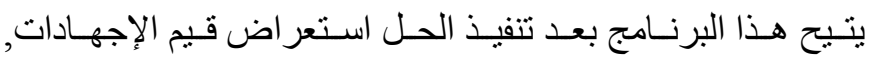
ومركبات الإزاحات, ورسومات توضيحية لتوزيع الإجهادات, و الإنحناءات و التشو هات الناتجة على النموذجات ورسو

و فيما يتعلق بإمكانيات برنامج الانسز في التنامس, فان البرنامج يجهز ثناثة أنو اع من نماذج التلامس وهي:

1 - Node - to - Node

2 - Node - to - Surface

3 - Surface - to - Surface

$$
\text { سقدة - عقدة - إلى - سطح - عقدة - إلى - سطح - سلى }
$$


و الذي يهمنا هو تلامس (Surface - to - Surface) و الذي تم اختياره وتطبيقه على النموذج المستخدم في هذا البحث.

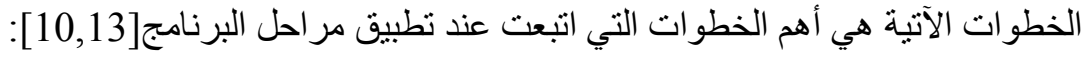
خو (I )

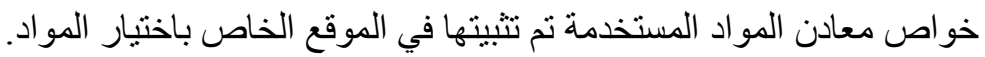

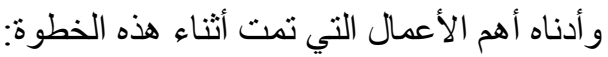

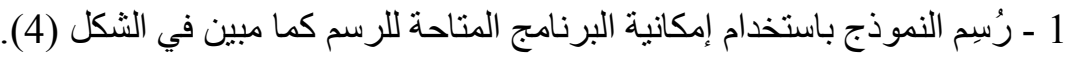

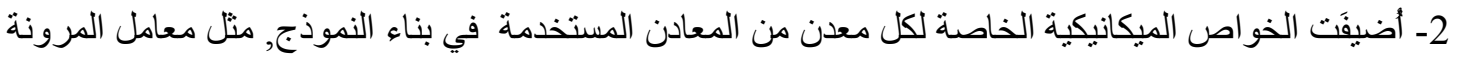
3 - نم اختيار نوع الثريحة الخاصة بهذا النموذج و هي (Structural Solid 185\&95).

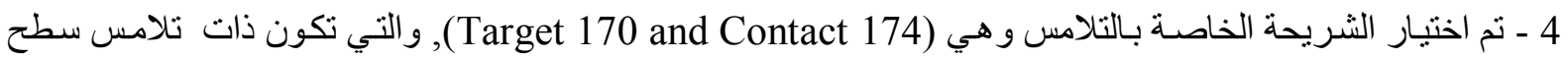
إلى سطح.

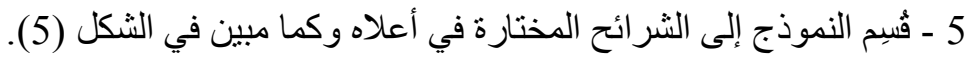
6 - أختير زوج التلامس (Contact Pair) Solution ( II )

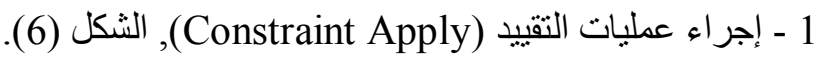
2 - إجراء عمليات تسليط الحمل (Load Apply) 3- إجر اء عمليات تتظيم عدد ألـ (Sub Steps), وكذلك (Load Step ( ألـ (Lod ). (Postprocessor ( III ) أو عند أي مرحلة. وبعد إنهاء وقت التنفيذ فإن جميع النتائج ولدى أي وقت يمكن أن يتم الحصول عليها.

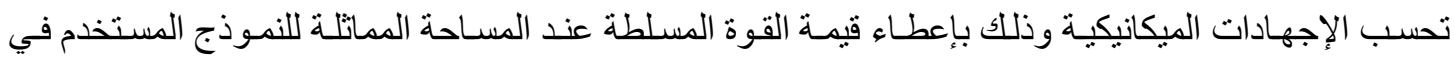
البرنامج.

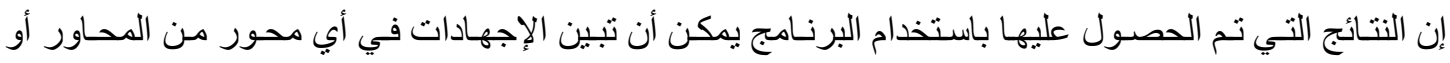

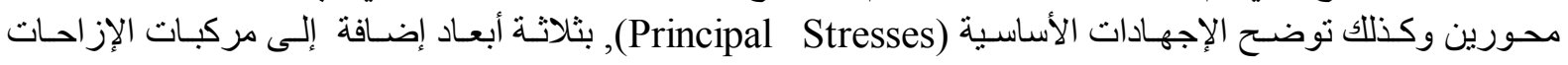

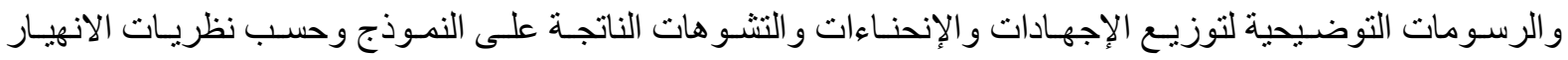

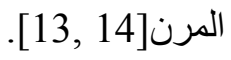
وأخير ا فان الفرضيات المتبعة في بناء نموذج العجلة الحاملة وعملية إيجاد قيم الإجهادات, فلقد اتبعت أدناه في عملية بنـاء

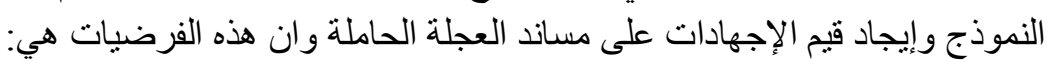

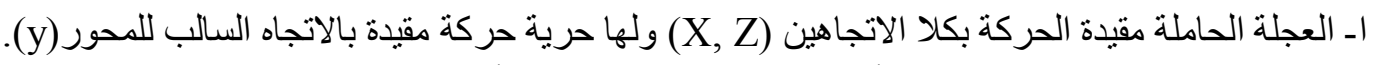

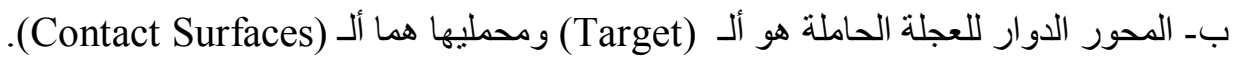
جـ- إهمال نأثير دوران الفرن أي اعتبار الفرن في طور التوقف إذ إن السرعة الدورانية للفرن قليلة ولا تتجاوز ألـ ( 1.2

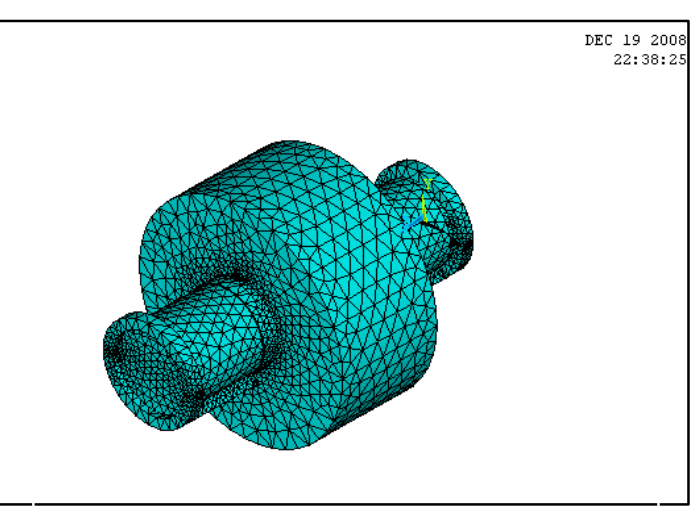

الثكل (4): النموذج المصمح للعجلة الحاملة باستخدام برنامج (ANSYS) (بل).

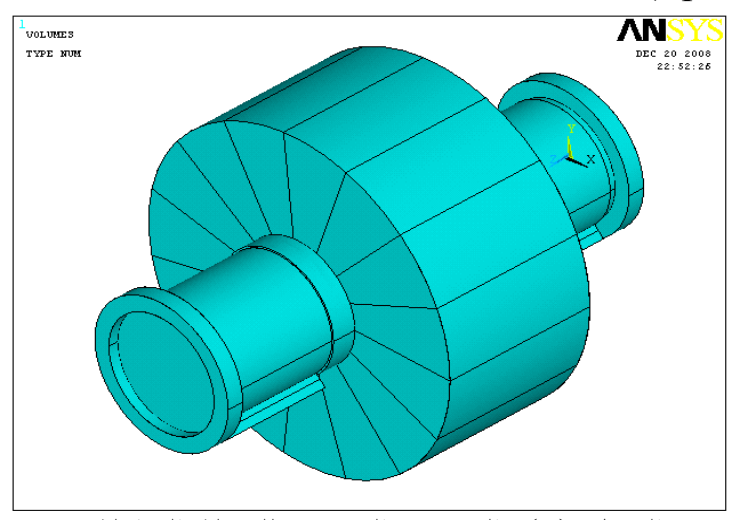

الثكل (5): النموذج المصمح للعجلة الحاملة

بعد تقسيمه إلى الثر ائح المختارة. 


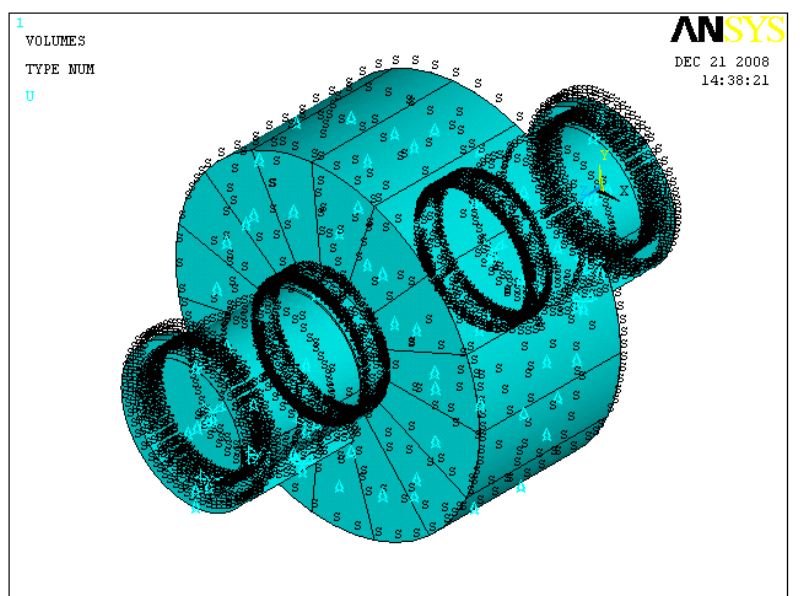

الثكل (6): النموذج بعد إجر اء عمليات التقييد.

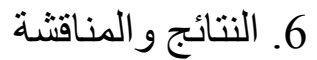

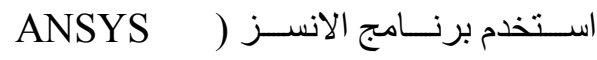

(Program V9.0

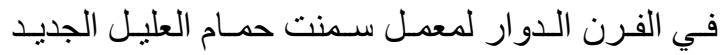

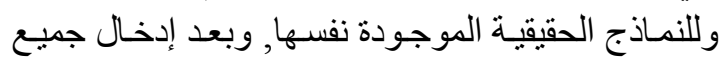

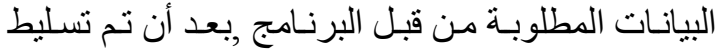

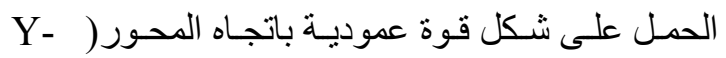

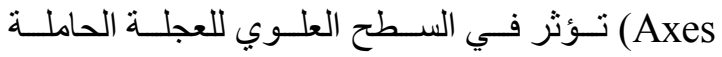

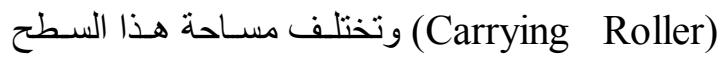

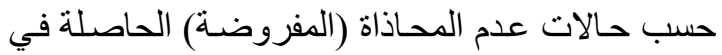

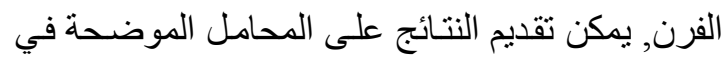

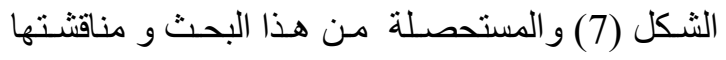
بتتـاول الرسومات و المنحنيات المعروضـة على النحو النحو

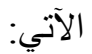

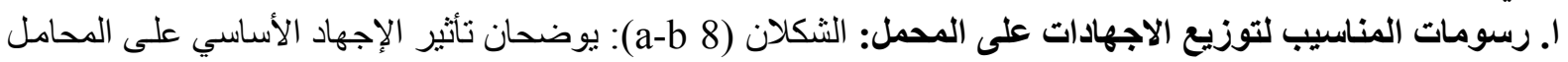

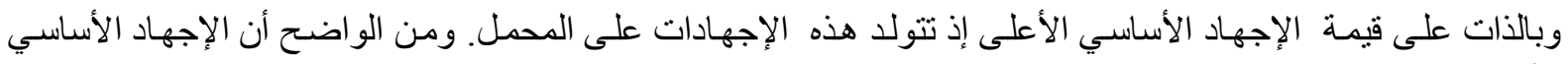

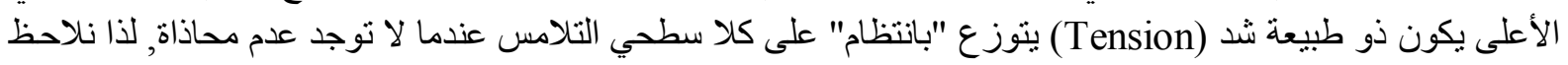

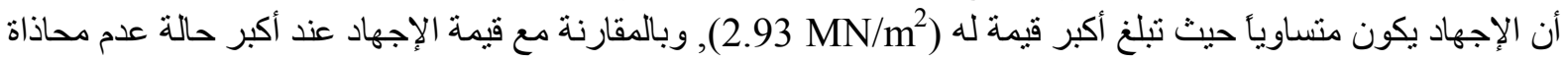

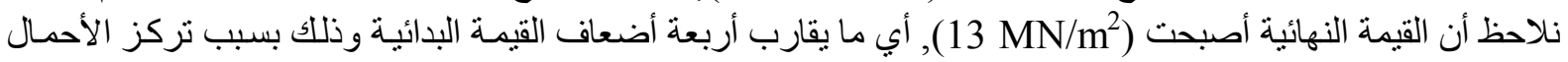

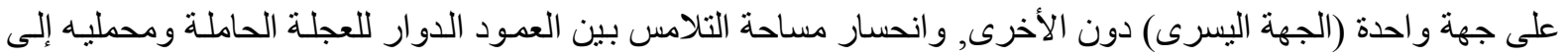

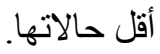
الثكلان (a-b 9): يوضحان نأثير تغير حالة عدم المحاذاة في قيمة الإجهاد باتجاه المحور(Y) إذ تنولد هذه الإجهادات

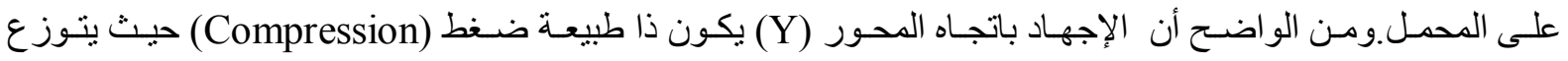

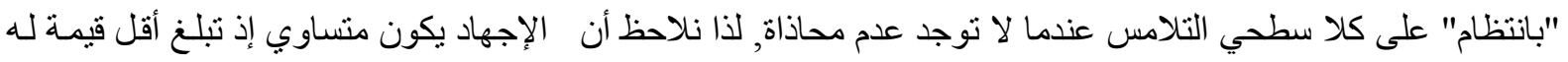

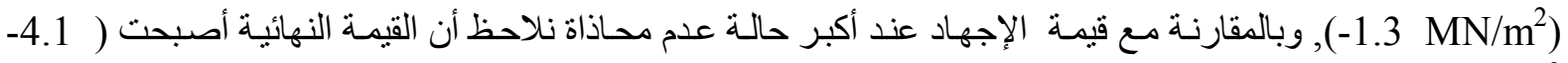

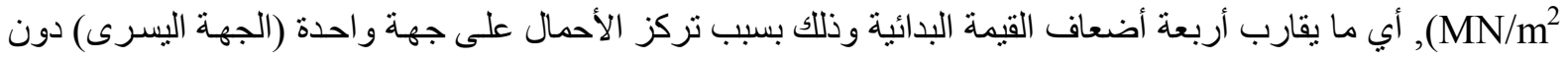

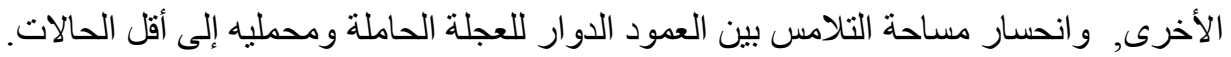

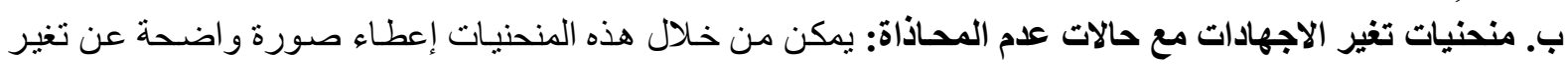

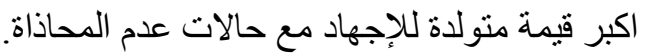

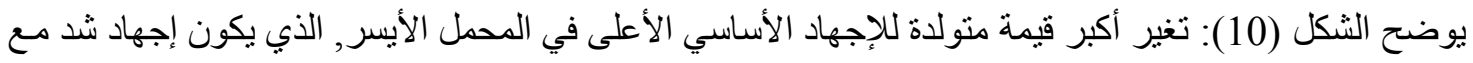

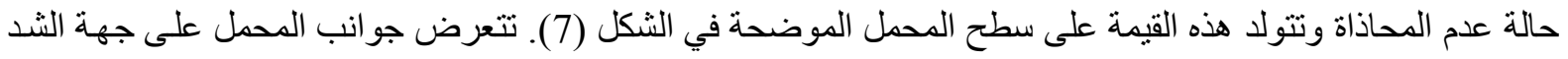

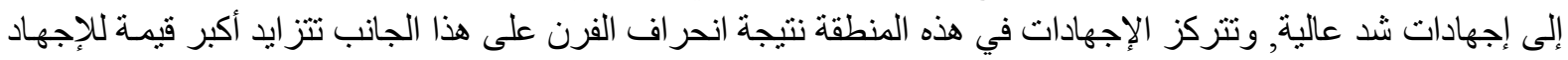
الأساسي الأعلى مع تز ايد حالة عدم المحاذاة.

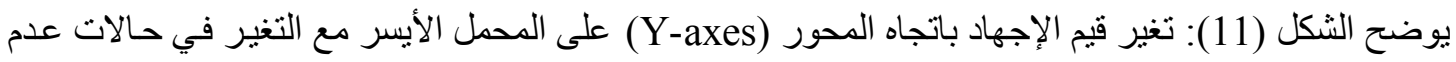

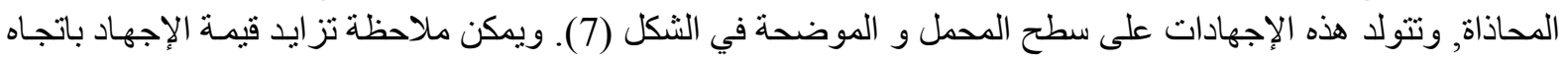

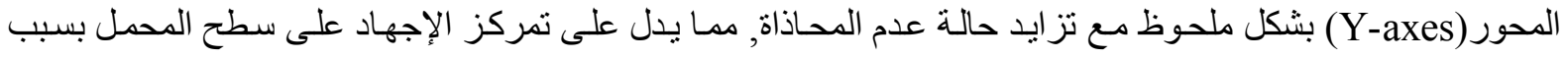
الحمل الناتج من انحر اف الفرن باتجاه هذا المحمل.

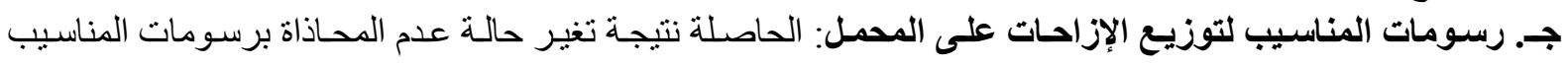

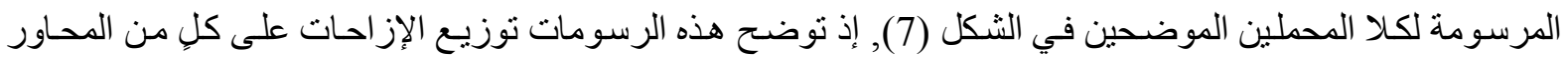

الثلاثة

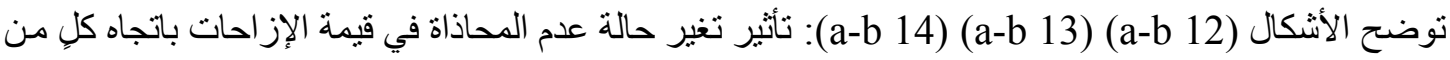
المحاور (X, , Y , Z) إذ تتولد هذه الإزاحات على المحمل. من الواضح أن هذه الإزاحات تتوزع "بانتظام" على سطحي 
التنلامس عندما لا توجد عدم محاذاة, لذا نلاحظ أنها متساوية على المحملين إذ تبلغ أقل قيمة لها و على التو الي

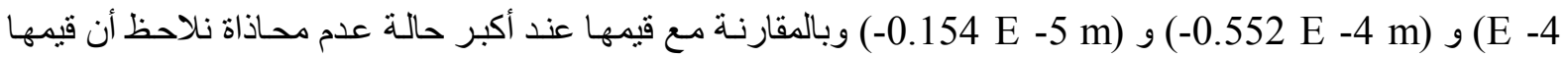

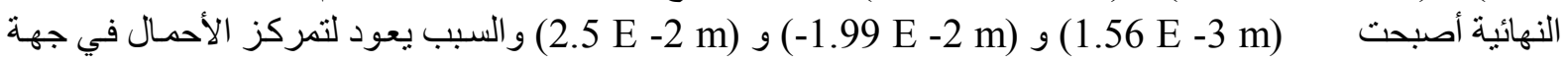

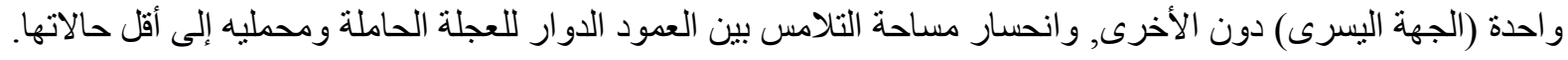

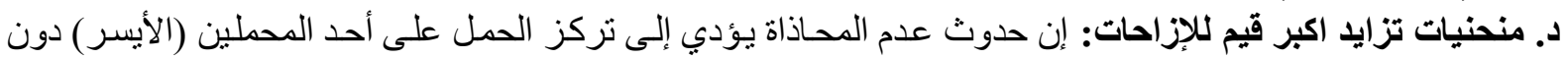

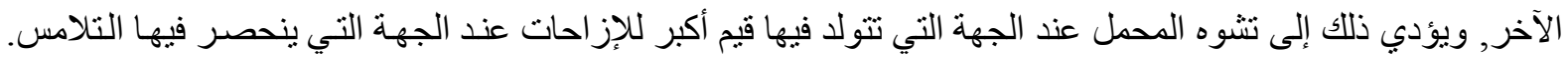

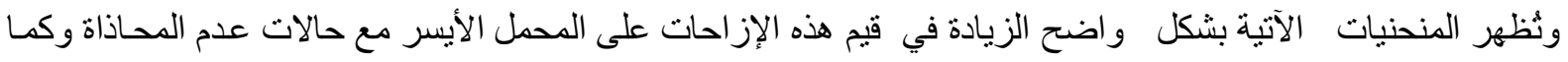

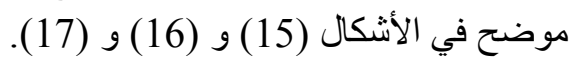
هو يوضح الثكل (15): تغير قيم الإزاحات باتجاه المحور (X) (X) على المحمل الأيسر مـع التغير في حالات عدم المحاذاة,

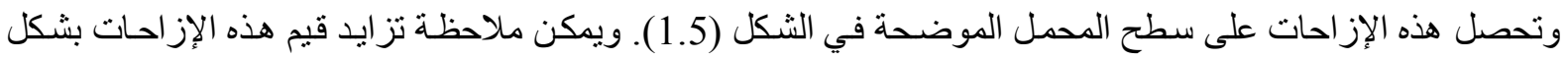

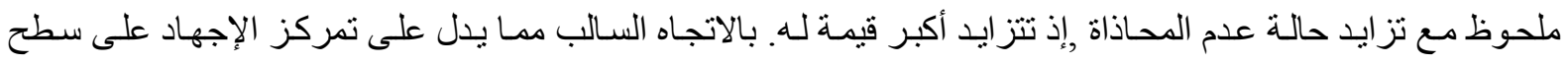

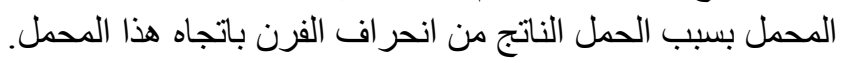

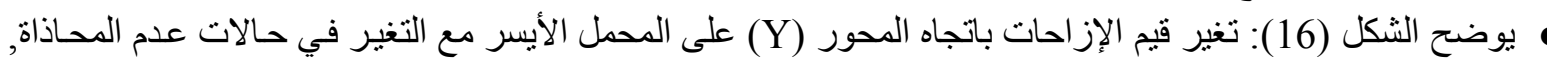

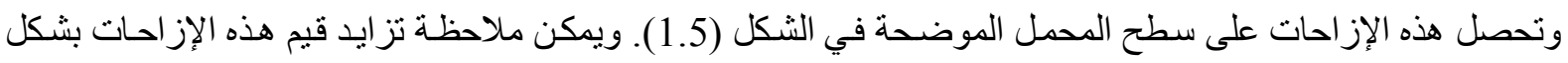

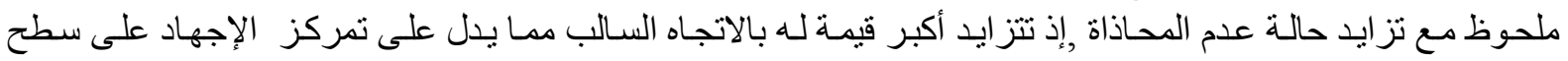

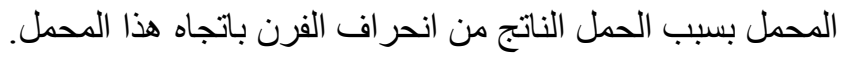

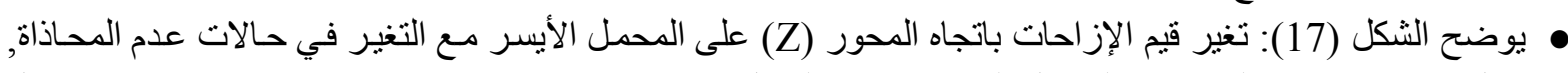

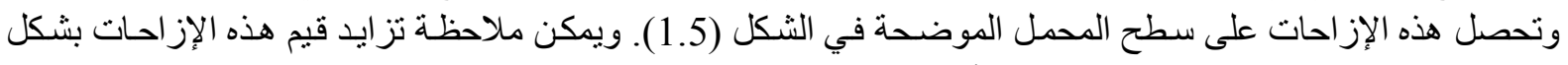

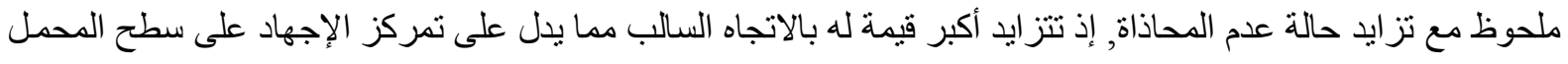
بسبب الحمل الناتج من انحر اف الفرن باتجاه هذا الدمعل.

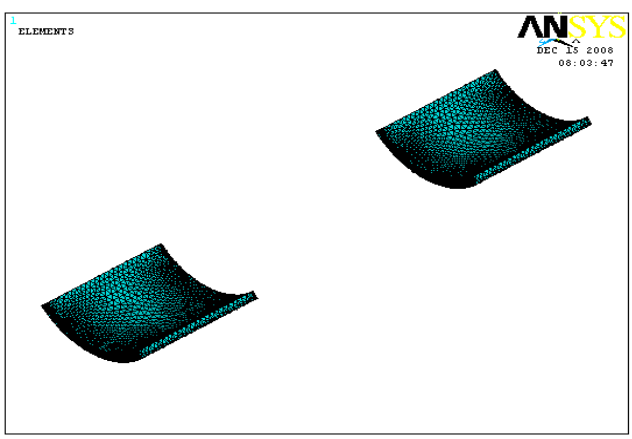

الثكل (7): مِحملا العجلة الحاملة.

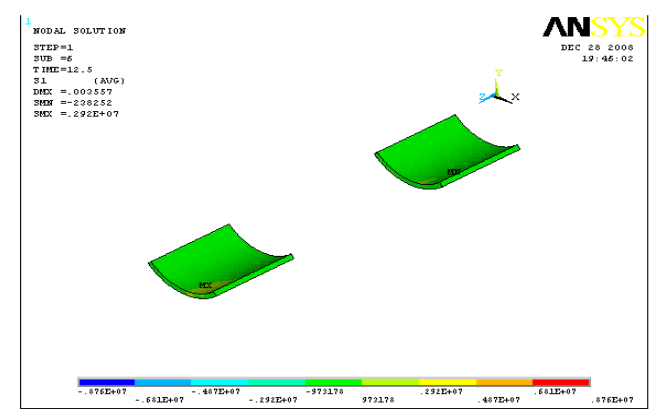

a: (Case 1) : 100\%

(لا نوجد عدم محاذاة)

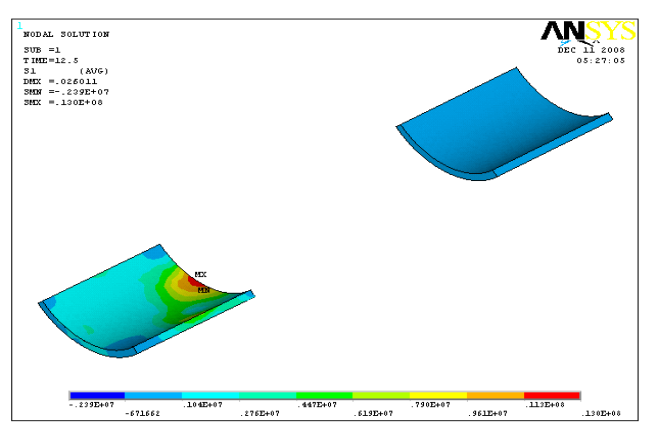

b: (Case 6): $50 \%$

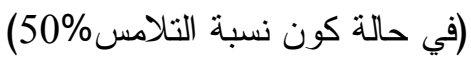

الثكل (8): توزيع الإجهاد الأساسي الأعلى على المحامل. 


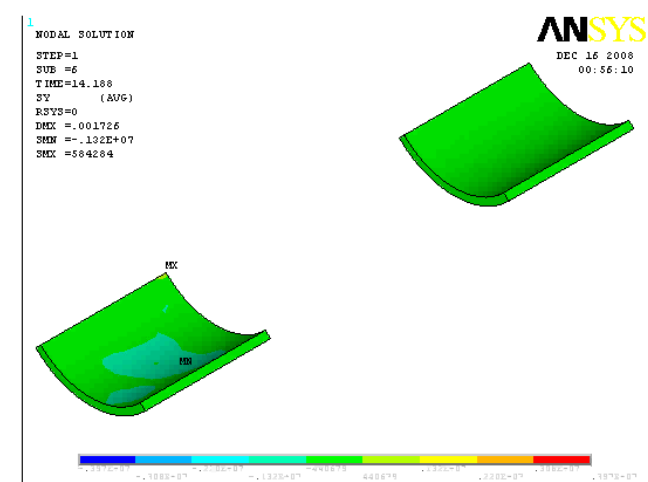

a: (Case 1): 100\%

(لا توجد عدم محاذاة)

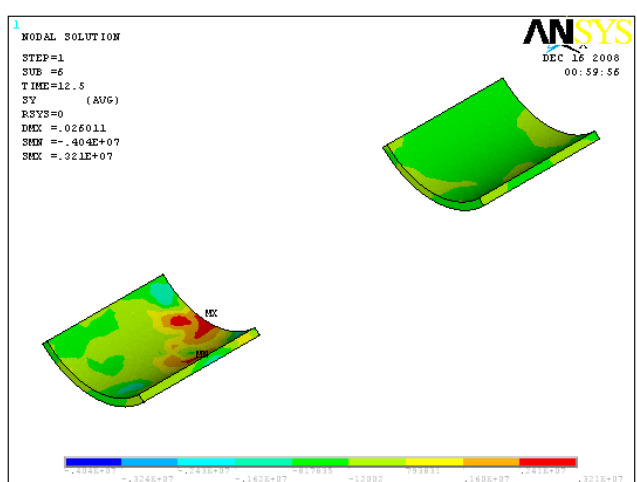

b: (Case 6): 50\%

(فى حالة كون نسبة التلامس\%) (Case :)

الثكل (9): توزيع الإجهاد باتجاه المحور (Y) على المحامل.

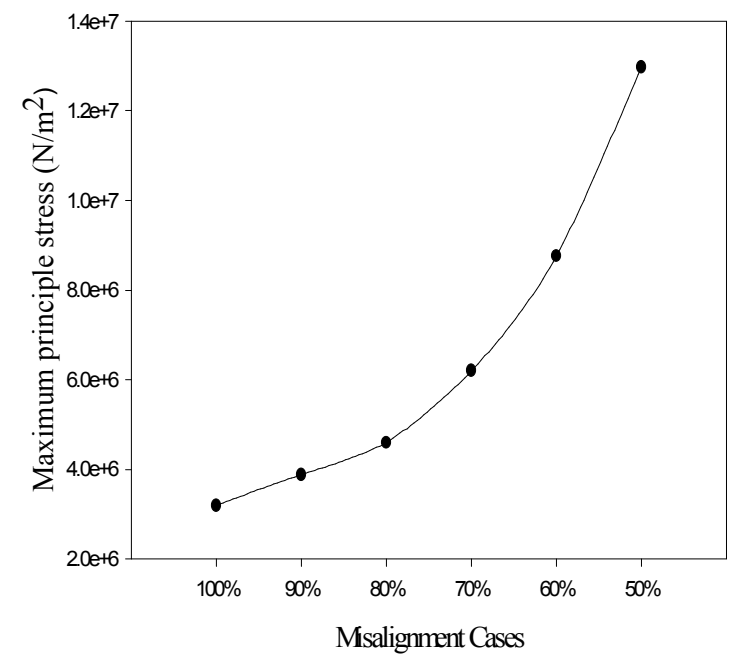

الثكل (10): تغير قيم الإجهاد الأساسـي الأعلــى للمحمل الأيسر مع حالات عدم المحاذاة.

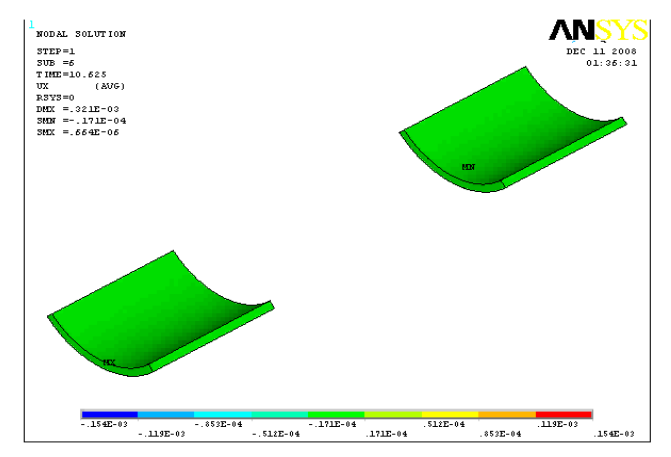

a: Case (1): 100\%

(لا توجد عدم محاذاة)

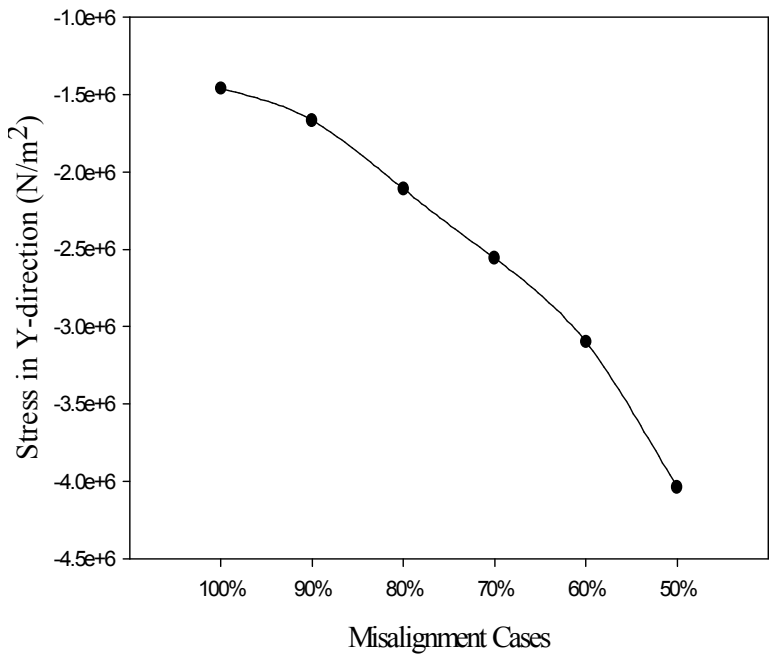

الثكل (11): تغير قيم الإجهاد علــى المـــور (Y) للمحمل الأيسر مع حالات عدم المحاذاة.

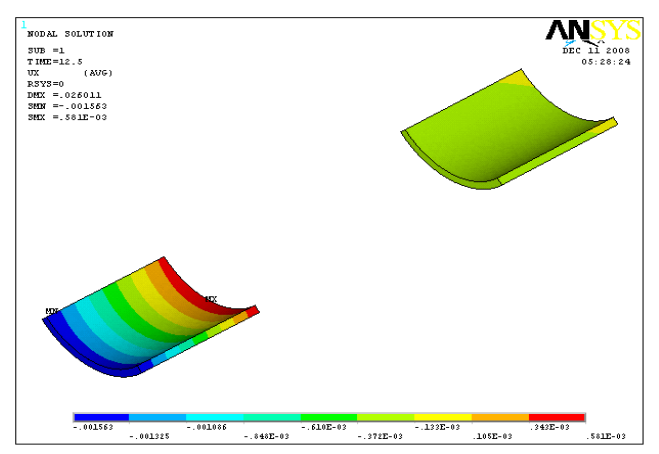

b: (Case 6): 50\%

(في حالة كون نسبة التلامس $50 \%$ (Case : 
الثكل (12): توزيع الإز احات على المحامل باتجاه المحور (X).

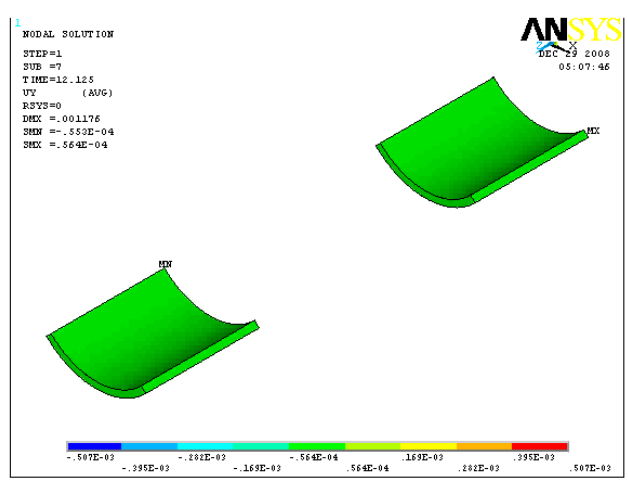

a: Case (1): 100\%

(لا توجد عدم محاذاة)

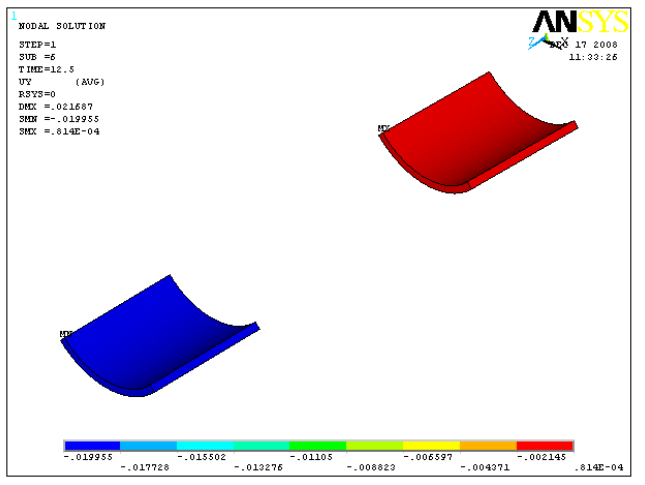

b: (Case 6): $50 \%$

(في، حالة كو ن نسبة التلامس\%

الثكل (13): توزيع الإز احات على المحامل باتجاه المحور (Y).

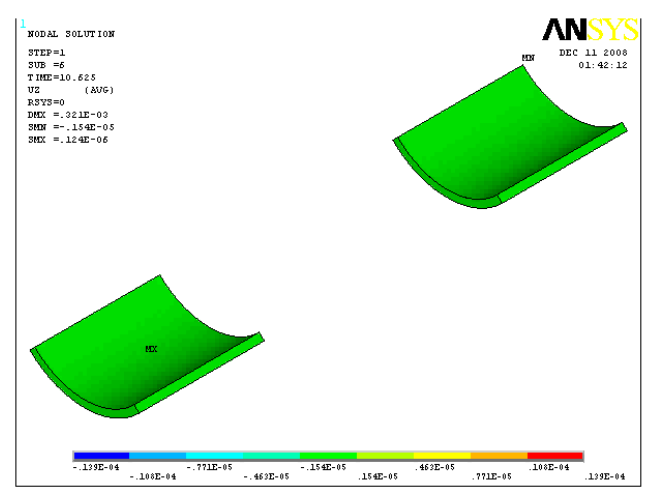

a: Case (1): 100\%

(لا تو جد عدم محاذاة)

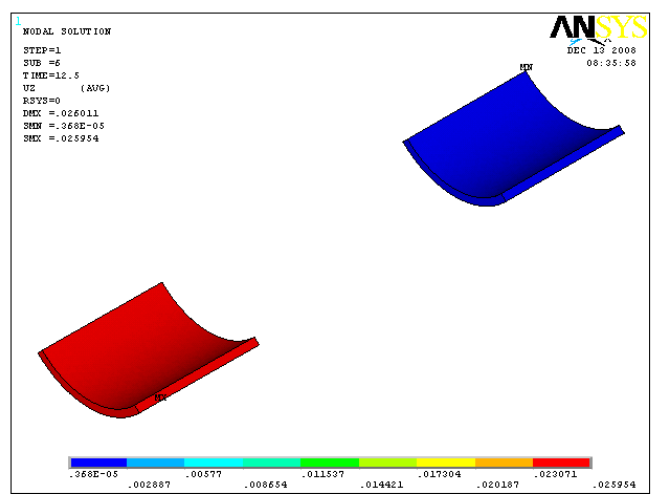

b: (Case 6): 50\%

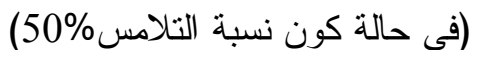

الثكل (14): توزيع الإزاحات على المحامل باتجاه المحور (Z).
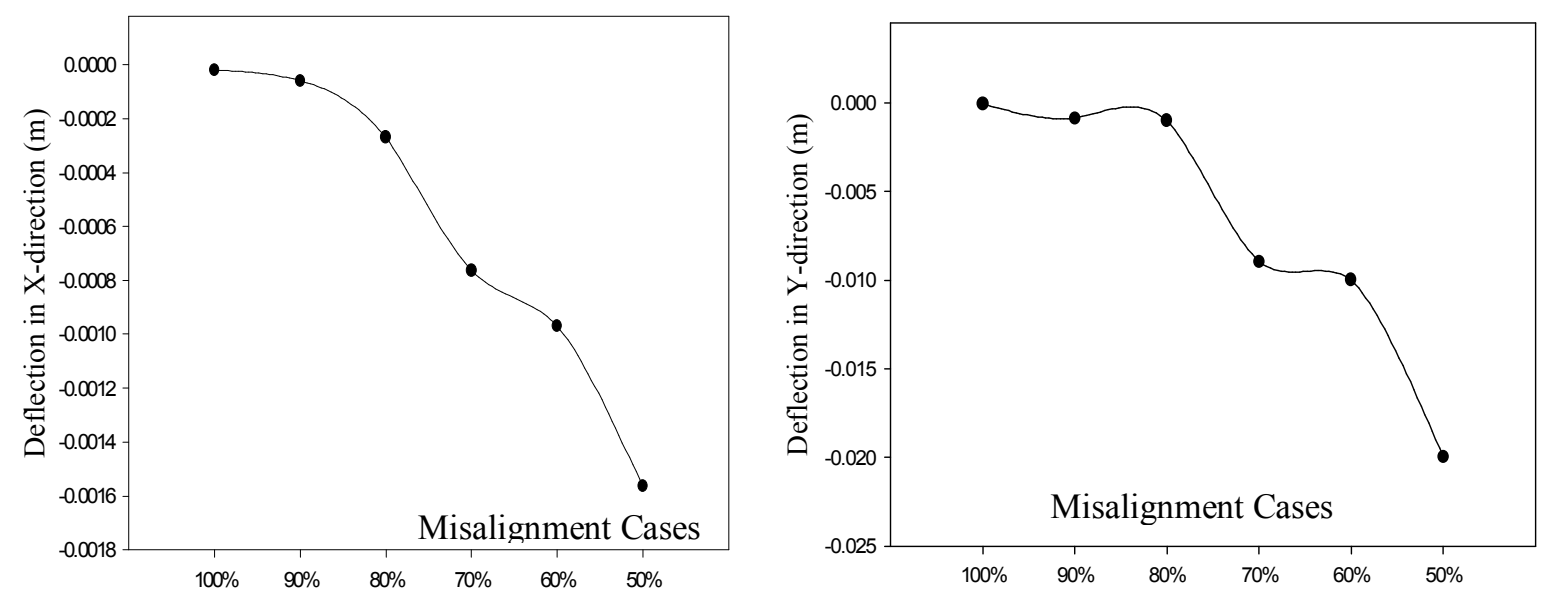

الثكل (15): تغير قيم الإز احات للمحمل الأيسر الثكل (16): تغير قيم الإزاحات للمحمل الأيسـر باتجاه المحور (X) مع حالات عدم المحاذاة. 


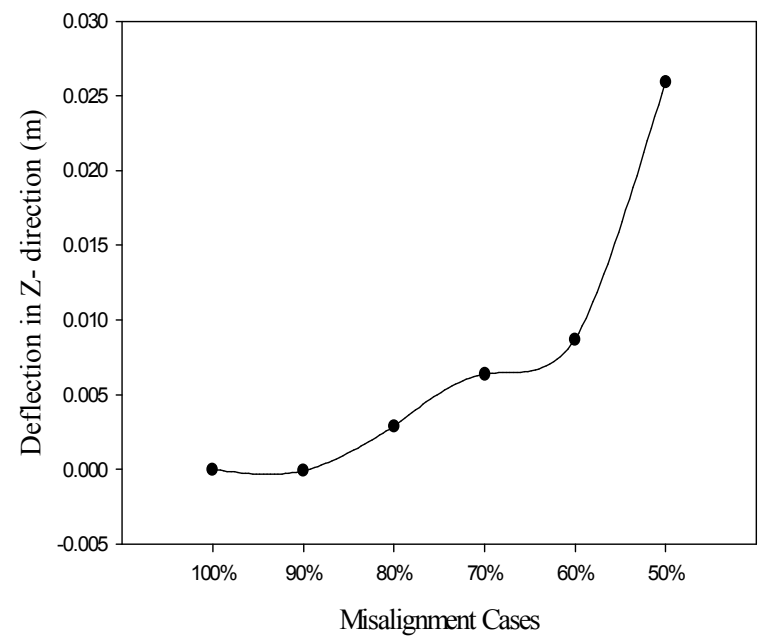

الثكل (17): تغير قيم الإزاحات للمحمل الأيسر باتجاه المحور (Z) مع حالات عدم المحاذاة.

7. الاستنتاجات يمكن اسنتناج النقاط التالية: 1- إن عدم المحاذاة له تأثير كبير في قيم الإجهادات على النى

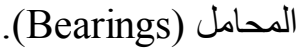

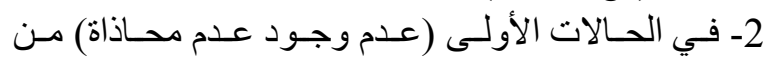
حالات عدم المحاذاة تكون ذات تأثير قليل نسبيا، إذ نلاحظ

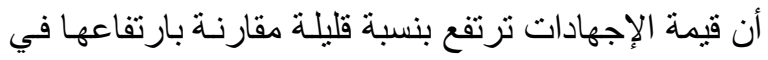
الحالات الأخيرة(في حالة كون نسبة التلامس

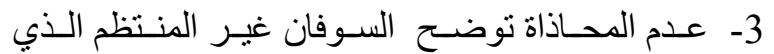

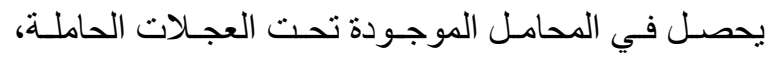

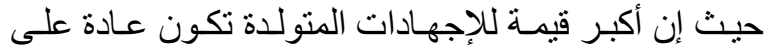

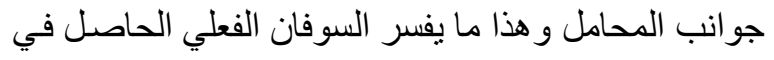

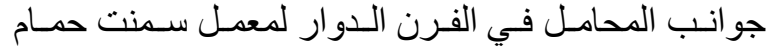

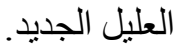
4- إن زيادة الإجهادات في جهة معينـة (محمل من محامل

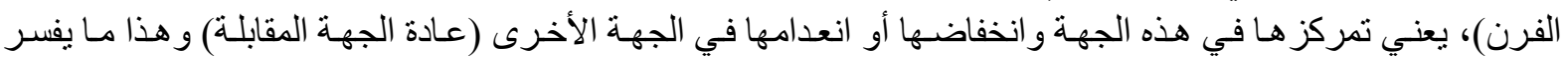

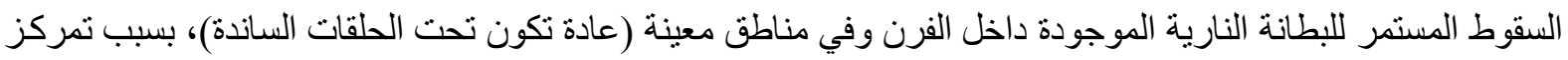
الإجهادات فيها مما يؤدي إلى تشوه لان لهذه المنطقة.

المصادر

1. Cement Manufacturing Courses (ASEC), "kilns and Preheater systems course", Egypt, (2005).

2. سلطان جمال نايف ,مجيد سعد عز الدين."حساب وزن و استقامة الفرن الدوار في معمل سمنت حمام العليل الجديد و اقع والع

حال الفرن", مكتب الخدمات الفنية والهندسية في المعهد التقني في الموصل ونلف (2003).

3. Warner I., "Rotary Kiln Loading, Rock Products", (1953).

4. Seaman W. C., "Chemical Engineering Process", (1951).

5. Bayard R. A., "Chemical and Metallurgical Engineering", (1953).

6. FLSmidth Company ,"Crank and Ovality Inspection of Kilns ", FLSmidth Company Parts and Service Division, (2001).

7. Coz Diaz. J.J, Rodriguez F., Garcia P.J., "Design a Finite Element Analysis of a Wet Cycle Cement Rotary Kiln" University of Oviedo, Spain ( 2001).

8. Saeed M. M, "Stress Analysis and Adjustment of a Rotary Kiln in The New Hammam AlAllel Cement Company", M.Sc Thesis. University of Mosul, Iraq (2006).

9. Staad Pro 2004 Online Manual and Help.

10. Help of The ANSYS Program V9.0.

11. Heran E. J., "Mechanics of Materials", Pergamon International Library, (1997).

12. Tahir H. T., "Mechanical Design of Moldboard Plow Bottom Throe Stresses Analysis and Performance Measurement", Ph.D. Thesis. University of Mosul, Iraq (2004).

13. Najim M. K., "Reduction of Thermal Stresses in Spur Gear Using Axial and Radial Holes" , Ph.D. Thesis Submitted. University of Mosul, Iraq (2008).

\section{تم اجراء البحث في كلية ألهنذسة = جامعة ألموصل}

